\title{
Difficult airway in an infant with fetal valproate syndrome
}

\author{
Eiko Onishi $\cdot$ Hitoshi Ishii $\cdot$ Chikanobu Sasaki
}

Received: 28 August 2013/Accepted: 4 November 2013/Published online: 1 December 2013

(C) Japanese Society of Anesthesiologists 2013

Keywords Laryngeal web - Fetal valproate syndrome $\cdot$ Difficult airway

To the Editor:

Gestational exposure to valproic acid can cause fetal valproate syndrome (FVS), characterized by a typical facial appearance and major congenital malformations (Supplemental Table 1) [1]. We report unexpected airway stenosis caused by a laryngeal web with FVS.

A male FVS infant was scheduled for surgery to repair a left inguinal hernia 2 months after delivery (weight $3,144 \mathrm{~g}$, height $48 \mathrm{~cm}, 41$ weeks of corrected gestation). General anesthesia was induced with nitrous oxide, oxygen, and sevoflurane without a muscle relaxant. Tracheal intubation was attempted, and multiple endotracheal tubes (2.0-3.0 mm internal diameter uncuffed) could not be advanced beyond the vocal cords. Laryngoscopic examination showed a pinhole stenosis of the trachea as a result of an anterior laryngeal web (Supplemental Fig. 1a), and resistance was felt at the level of the vocal cords. Insertion of the scope with gentle pressure detached the laryngeal web, and the rigid bronchoscope could be advanced beyond the vocal cords. Slight hemorrhage of the anterior

Electronic supplementary material The online version of this article (doi:10.1007/s00540-013-1750-1) contains supplementary material, which is available to authorized users.

E. Onishi $(\varangle) \cdot$ H. Ishii · C. Sasaki

Department of Anesthesiology, Sendai Red Cross Hospital,

2-43-3 Yagiyama honcho, Taihaku-ku, Sendai,

Miyagi 982-8501, Japan

e-mail: e-came78@jk9.so-net.ne.jp commissure was observed (Supplemental Fig. 1b). Surgery was postponed with consideration for glottic edema caused by the vocal cord injury. An examination of the larynx 6 days after was normal. Surgery was performed 2 weeks later. The trachea could be intubated with a $2.5-\mathrm{mm}$ tube without resistance.

Congenital webs result from incomplete recanalization of the primitive larynx. Airway stenosis with FVS caused by a laryngeal web has not been reported. However, it may possibly be one of the anomalies associated with FVS. Our patient remained asymptomatic until the unexpected finding that intubation is difficult. Difficult intubation during general anesthesia is a life-threatening complication. Therefore, preoperative airway examination should be diagnostic when an infant with FVS needs surgery under general anesthesia.

\section{Reference}

1. Clayton-Smith J, Donnai D. Fetal valproate syndrome. J Med Genet. 1995;32:724-7. 